\title{
Envolvimento de alunos com TEA em situações de bullying de acordo com múltiplos informantes
}

\author{
Carla Samya Nogueira Falcã $0^{1}$ \\ ORCID: 0000-0001-6772-3199 \\ Ana Carina Stelko-Pereira ${ }^{2}$ \\ ORCID: 0000-0002-8089-132X \\ Dayse Lôrrane Gonçalves Alves ${ }^{3}$ \\ ORCID: 0000-0002-8439-1066
}

\section{Resumo}

Há lacuna de estudos nacionais acerca de pessoas com Transtorno do Espectro Autista (TEA) e envolvimento em bullying escolar, ainda que esses indivíduos frequentem cada vez mais escolas regulares. Assim, este estudo identificou a participação em bullying de estudantes com TEA, de acordo com eles mesmos, seus pais e seus professores de educação física (EF), bem como analisou o quanto conhecem a respeito do fenômeno. Trata-se de um estudo transversal, com abordagem analítica. A amostra foi de 133 indivíduos, 63 pais (97\% do sexo feminino), 51 professores de EF (56,9\% homens e 78\% lecionando em escolas públicas) e 19 alunos com TEA (18 do sexo masculino). Os participantes foram recrutados em uma instituição que faz atendimentos especializados em Fortaleza, Ceará. Foram aplicadas entrevistas estruturadas de acordo com cada categoria de participante. Os professores de EF demonstraram maior entendimento sobre o bullying do que pais e estudantes, porém houve cinco professores que minimizaram o problema caracterizando-o como brincadeira entre crianças. Verificou-se que 30 pais $(47,6 \%)$ disseram não saber se o filho era envolvido em bullying e que 33 (52,4\%) identificaram o envolvimento, sendo $76 \%$ como vítima, 21\% como agressor/vítima e 3\% como agressor. Já os professores afırmaram que 53\% dos estudantes com TEA não estavam envolvidos no problema e os outros 47\% apontaram-nos como vítima. Por fim, 63\% dos alunos declararam-se vítimas. Essas diferenças foram estatisticamente relevantes. Portanto, urge a necessidade de programas educativos para toda a comunidade escolar com o intuito de prevenir o bullying e promover uma inclusão escolar efetiva dos estudantes com TEA.

\section{Palavras-chave}

Bullying - Transtorno do Espectro Autista - Escolas regulares - Inclusão escolar.

1 - Universidade Regional do Cariri (URCA), Iguatu, Ceará, Brasil. Contato: ksamyaf@hotmail.com

2- Universidade Federal do Paraná (UFPR), Curitiba, Paraná, Brasil. Contato: anastelko@gmail.com

3 - Universidade Estadual do Ceará (UECE), Fortaleza, Ceará, Brasil. Contato: dayselorranealves@gmail.com

(i) (\&) https://doi.org/10.1590/S1678-4634202147217359

BY NC This content is licensed under a Creative Commons attribution-type BY-NC. 


\section{Involvement of students with ASD in bullying according to multiple informants}

\section{Abstract}

There are few studies on people diagnosed with Autism Spectrum Disorder (ASD) and their involvement in school bullying in Brazil, especially because many of these children have been enrolled in regular schools. In this cross-sectional analytical study, we investigate how students with ASD experienced bullying situations, according to themselves, their parents and their Physical Education (PE) teachers. We also investigate how much they know about this phenomenon. We interviewed a total of 133 individuals, 63 parents (97\% female), 51 PE teachers (56.9\% male and 78\% public school teachers) and 19 students with ASD (18 male). We selected the participants from a special education institution in Fortaleza, Ceará. Our team performed structured interviews according to each category of participant. PE teachers showed a greater understanding of bullying than parents and students, but five teachers naturalized the problem by calling it "roughhousing". A total of 30 parents $(47.6 \%)$ claimed not to know whether the child had experienced bullying, while 33 (52.4\%) were aware; 76\% identified the child as a victim, 21\% as a bully-victim, and $3 \%$ as a bully. According to the teachers, 53\% of students with ASD were not involved in bullying situations, while $47 \%$ of teachers reported that special-needs children were victims. Finally, 63\% of the students declared themselves victims. These differences were statistically relevant. Our results indicate that there is a need for educational programs for the entire school community in order to prevent bullying and promote the effective inclusion of students with ASD.

\section{Keywords}

Bullying - Autism Spectrum Disorder - Regular Schools - Inclusion.

\section{Introdução}

0 bullying no ambiente das escolas é uma grave manifestação de violência entre estudantes que traz prejuízos ao desenvolvimento saudável de crianças e adolescentes. Esse fenômeno define-se pela ocorrência de agressões, repetidas e intencionais, emitidas por escolares em relação a outros, as quais têm o intuito de gerar sofrimento, dor e angústia. As referidas agressões podem ser físicas, psicológicas, verbais e/ou sexuais e ocorrem de forma direta ou indireta. 0 bullying direto efetiva-se quando as provocações são explícitas e imediatas entre agressor(es) e vítima(s). Já o bullying indireto ou relacional acontece de forma mais sutil, por meio de exclusão, isolamento social ou pela perpetuação de rumores e intrigas. Nas práticas de bullying, os estudantes podem estar envolvidos como autores, vítimas, autores-vítimas ou testemunhas (OLWEUS, 2013; RETTEW; PAWLOWSKI, 2016). 
Adicionalmente, o bullying escolar materializa-se em uma relação desigual de poder entre agressores e vítimas, pois essas, geralmente, têm dificuldades para defender-se das provocações (OLWEUS, 2013; RETTEW; PAWLOWSKI, 2016). A presente situação ocorre, porque a pessoa vitimizada pode apresentar um déficit de habilidades sociais em relação à assertividade, à defesa dos próprios direitos e à resolução de conflitos, bem como ter uma rede social limitada ou instável de amizades (GONZALEZ; RAMIREZ, 2017). Já o agressor pode tornar-se superior na relação de violência por apresentar maiores altura, idade ou força física, como também por ter seus comportamentos agressivos reforçados pelos pares (RETTEW; PAWLOWSKI, 2016). Outro fato que também torna as vítimas mais vulneráveis é a presença nas mesmas de traços físicos e/ou comportamentais visíveis e destoantes da norma social, como aparência e constituição corporal, baixo desempenho acadêmico ou comportamentos inadequados (MAÏANO et al., 2016a).

Revisões sistemáticas da literatura internacional demonstram que crianças e adolescentes que apresentam alguma ou múltiplas deficiências têm mais chances de serem vitimizados no ambiente escolar em relação aos demais estudantes (MAÏANO et al., 2016a; ROSE; MONDA-AMAYA; ESPELAGE, 2011). De forma mais específica, estudantes com Transtorno do Espectro Autista (TEA) encontram-se mais vulneráveis a envolverem-se em práticas de bullying, tanto como vítimas quanto como vítimas-agressores ou agressores, devido aos seus padrões comportamentais peculiares e aos seus severos déficits de habilidades sociais (HOOVER; KAUFMAN, 2018; HUMPHREY; HEBRON, 2015; HWANG et al., 2018; MAÏANO et al., 2016b; SCHROEDER et al., 2014; SRECKOVIC; BRUNSTING; ABLE, 2014). Além disso, estudantes com TEA ou com Transtorno do Déficit de Atenção com Hiperatividade (TDAH) são, provavelmente, mais vitimizados que estudantes com outros tipos de transtornos ou deficiências (HUMPHREY; HEBRON, 2015; SRECKOVIC et al., 2014).

0 TEA é definido como um transtorno do neurodesenvolvimento que se caracteriza por déficits na comunicação e na interação social, bem como pela presença de comportamentos restritos, repetitivos e estereotipados, sendo multideterminado por causas genéticas e ambientais (DEMETRIOU et al., 2018; WANG; KERY; XIONG, 2018). Com base em estudos epidemiológicos realizados nos últimos 50 anos, a Organização Mundial de Saúde (OMS) aponta que a prevalência de TEA parece estar aumentando globalmente. Essa organização ainda afirma que o TEA afeta uma em cada 160 crianças no mundo e que as pessoas com esse transtorno são frequentemente sujeitas à estigmatização, discriminação e violações de direitos (OPAS; OMS, 2017). Já no Brasil, estima-se que existam mais de dois milhões de pessoas com TEA, sendo 120 a 200 mil menores de cinco anos e 400 a 600 mil com idade inferior a 20 anos (IBGE, 2010).

Em revisão sistemática realizada em 2018, os pesquisadores descobriram que as crianças com TEA são intimidadas pelos pares de três a quatro vezes mais que os colegas sem o transtorno, o que gera impactos negativos para o funcionamento e para a saúde mental desses indivíduos, que têm o risco de suicídio aumentado pelas agressões recorrentes (HOOVER; KAUFMAN, 2018). Já em metanálise realizada por Schroeder et al. (2014), destacou-se que estudantes com TEA sofrem mais bullying nas escolas regulares do que em escolas especiais, além de tenderem a ser marginalizados e excluídos entre pares. Dessa forma, a prática do bullying nas escolas regulares não é coerente com a 
proposta de inclusão escolar de indivíduos com trantornos ou deficiências, pois as crianças e adolescentes têm seus direitos violados, são isolados socialmente e não participam ativamente do cotidiano escolar.

A Lei de Diretrizes e Bases da Educação Nacional (BRASIL, 2018) defıne Educação Especial como a modalidade de educação oferecida preferencialmente na rede regular de ensino, para educandos com deficiência, transtornos globais do desenvolvimento e altas habilidades ou superdotação. Assim, é dever das instituições educacionais regulares garantirem o acesso qualificado e efetivo desses indivíduos às atividades escolares.

Kassar e Rebelo (2018) apontam que essa visão de educação especial, a partir da perspectiva da educação inclusiva, fortaleceu-se entre 1974 a 2014 no Brasil, o que é demonstrado pelo aumento das matrículas desse público em escolas regulares, em paralelo à diminuição das classes e escolas especiais, principalmente, a partir da implementação da Política Nacional de Educação Especial na Perspectiva da Educação Inclusiva (PNEEPEI), de 2008 (BRASIL, 2008). Cabe mencionar que essa PNEEPEI tem sido considerada um grande avanço na concretização dos direitos das pessoas com deficiência. Portanto, justificam-se aumentos nos investimentos para sua efetivação em todo o território nacional (GIROT0; POKER; VITTA, 2018) e resistência diante da conjuntura política atual de retrocesso quanto a políticas públicas inclusivas (PAGNI, 2019; KASSAR; REBELO; DE OLIVEIRA, 2019).

De forma específica, há a Política Nacional de Proteção dos Direitos da Pessoa com TEA, Lei 12.764 de 27 de dezembro de 2012 (BRASIL, 2012), a qual enfatiza a inclusão de indivíduos com esse transtorno em classes comuns de ensino regular. Em decorrência dessa Lei, destaca-se que, de 2017 a 2018, a matrícula de crianças com TEA na rede pública cresceu 37,27\% (INEP, 2018). Resultados positivos têm sido apontados com a inclusão dessas crianças, como um repensar práticas pedagógicas que sejam mais específicas às necessidades e interesses de cada aluno, inclusive aos sem deficiência (MEL0; SANTIAG0, 2018).

Mesmo com essa lei, a efetiva inclusão de crianças e adolescentes com TEA no ambiente das escolas regulares é ainda um desafio para a educação brasileira, pois apenas a presença em sala de aula não assegura a aprendizagem e a inclusão social desses indivíduos. É necessário adaptar os contéudos, formar adequadamente os professores, combater o bullying e elaborar avaliações diferenciadas (AGRIPINO-RAMOS; LEMOS; SALOMÃO, 2019; BAPTISTA, 2019).

Em revisão sistemática da literatura nacional e internacional a respeito desse tema (CABRAL; MARIN, 2017), identificou-se que os maiores desafios para a inclusão dos estudantes com TEA são: as dificuldades de comunicação desses estudantes, o desconhecimento das características de um indivíduo com TEA pelos professores e a carência de estratégias pedagógicas que impactam no processo de aprendizagem. Adiciona-se a isso a carência de profissionais de Atendimento Educacional Especializado (AEE) e de Salas de Recursos Multifuncionais, bem como pequena articulação entre o professor da turma e o da sala de recursos (PASIAN; MENDES; CIA, 2017; SANTOS et al., 2017). Assim, é fundamental que haja uma maior preocupação com a formação de professores, com a construção de ferramentas específıcas que facilitem o processo de aprendizagem e comunicação dos estudantes com TEA e com a efetiva implantação da PNEEPI (BRASIL, 2008). 
É escassa a literatura brasileira acerca do envolvimento de crianças com TEA em bullying, enquanto que já existem estudos internacionais sobre bullying e o envolvimento de crianças e jovens com TEA e que utilizaram de diversos informantes para coleta de dados (ADAMS et al., 2016; HEBRON; HUMPHREY; OLDFIELD, 2015; HWANG et al., 2018; UNG et al., 2016), principalmente pais, os próprios indivíduos com TEA, os seus colegas e professores. Assim, cabem os seguintes questionamentos: estudantes com TEA de escolas regulares brasileiras envolvem-se em bullying, de acordo com eles mesmos, seus pais e professores de educação física? Se sim, como ocorre esse envolvimento interpessoal e quais são as consequências percebidas?

Dessa forma, esta pesquisa objetiva identificar o envolvimento de estudantes com diagnóstico de TEA em bullying, de acordo com eles mesmos, seus pais e seus professores de educação física.

\section{Método}

Esta pesquisa foi aprovada por Comitê de Ética em Pesquisa da Universidade Estadual do Ceará (UECE), Parecer 1.691.480/2016, e se refere a um estudo transversal e com abordagem analítica. 0 estudo transversal é o delineamento que permite estabelecer informações acerca de um fenômeno, como dados socioeconômicos e/ou sociodemográficos, a partir de dados de uma amostra extraída em um momento determinado e de uma população bem delimitada (LOPES, 2017). Já os estudos analíticos avaliam as associações entre variáveis, de modo a contribuir para elucidar os determinantes de um problema (HULLEY et al., 2015).

Participaram desse estudo estudantes com TEA com idade entre 10 e 15 anos, que frequentavam uma instituição especializada, para apoio clínico e psicopedagógico e, paralelamente, também estavam matriculados em escolas regulares. Essa instituição é referência no cuidado a indivíduos com TEA; localiza-se em Fortaleza/CE e atende cerca de 400 estudantes da rede pública e privada, de todas as faixas etárias, realizando atividades como intervenção precoce, AEE, oficinas e vivências terapêuticas.

Após um levantamento na instituição, foram encontrados 66 alunos que se encaixavam no perfil desta pesquisa. No entanto, apenas 19 alunos tinham habilidades comunicativas para responder ao questionário do estudo. Desses, 14 alunos eram de escolas públicas e cinco de escolas privadas, sendo 18 do sexo masculino e um do sexo feminino. Dos pais/cuidadores dos 66 alunos, 63, espontaneamente, aceitaram participar desta pesquisa, sendo que 61 eram do sexo feminino e dois do sexo masculino.

Os professores de educação física (EF) participantes da pesquisa também foram eleitos de acordo com os 66 alunos com TEA da instituição especializada que se encaixavam no perfil da investigação. Quatro professores davam aulas a mais de uma criança com TEA participante do estudo, dois estavam de licença médica e seis davam aulas de EF, porém não tinham formação específica. Desse modo, participaram 51 professores de EF, os quais responderam questionários referentes a 56 alunos com TEA, sendo 40 professores de escolas regulares públicas e 10 de escolas privadas do Estado do Ceará. Ressalta-se que um (1) professor lecionava em mais de uma escola, o que explica o quantitativo de escolas 
ser menor que o de professores. Para este estudo, foram escolhidos, especificamente, os professores dessa área, pois as aulas que envolvem jogos e práticas corporais são propícias à ocorrência do bullying (O'CONNOR; GRABER, 2014; HWANG et al., 2018).

A coleta de dados ocorreu por meio da aplicação de entrevistas estruturadas, sendo que todos assinaram Termo de Consentimento Livre e Esclarecido quando maiores de 18 anos ou Termo de Assentimento, no caso dos alunos. As entrevistas com pais e/ou cuidadores e professores foram realizadas pela primeira pesquisadora deste artigo e por dois auxiliares de pesquisa, formados em Pedagogia. Os entrevistadores leram item a item junto aos participantes, esclarecendo dúvidas e anotando as respostas. Já a entrevista com os estudantes com TEA foi aplicada pelo próprio profissional da instituição especializada, pois ele realizava atendimentos semanais com esses indivíduos. Todas as entrevistas seguiram roteiros que foram elaborados pelas duas primeiras autoras deste artigo.

Com as pessoas com TEA, empregou-se um roteiro de entrevista estruturada com questões de identificação sociodemográfica. Em seguida, solicitou-se que o aluno dissesse o que é bullying. Quando necessário, foi explicado o que é bullying ao respondente. Então, questionou-se se o entrevistado foi vítima de bullying nos últimos seis meses, caso sim, solicitou-se que relatasse o ocorrido. Por fim, inquiriu-se se o aluno praticou bullying.

Entrevistaram-se os pais e cuidadores de crianças com TEA com o auxílio de um roteiro composto por perguntas acerca de identificação sociodemográfica e seis questões especificas a respeito de bullying. Dessas seis questões, duas foram abertas e referiam-se à definição de bullying e a procedimentos adotados pelos pais diante da desconfiança do envolvimento do filho em bullying. As demais foram fechadas e tratavam do envolvimento em bullying nos últimos seis meses: se o filho era vítima, agressor ou vítima/agressor; quais as consequências decorrentes do envolvimento; e reações ao descobrirem que seus filhos estão envolvidos em situação de bullying.

Já com os professores de EF, aplicou-se um roteiro de entrevista com itens sobre identificação sociodemográfica e seis perguntas em relação ao fenômeno. Dessas seis, a primeira pergunta versava a respeito do conhecimento que o professor tinha sobre $o$ tema bullying. Já as questões seguintes, dois, três e quatro, solicitavam ao professor que relacionasse os nomes de alunos da turma, na qual lecionava e da qual a criança com TEA fazia parte, que estivessem envolvidos em situações de bullying. Assim, o professor não sabia, no início da entrevista, que o estudo se referia a investigar, especificamente, a relação entre o bullying e os alunos com TEA. 0 professor apenas respondia a quinta questão, a qual tratava das características do aluno com TEA que o tornam mais vulnerável ao bullying, caso mencionasse algum aluno com esse transtorno. Se o professor não mencionasse o aluno com TEA como envolvido em bullying, respondia ao seguinte item: "Você não mencionou o(s) aluno(s) com TEA. Em sua opinião, como é o relacionamento interpessoal do aluno com os colegas?".

Após a coleta de dados, empregou-se, para o processo de tabulação das questões fechadas, o programa estatístico Statistical Package for Social Science (SPSS) da IBM (International Business Machines Corporation) 2.0. As respostas foram analisadas descritivamente e foram comparadas as variáveis conhecimento sobre bullying e tipo de envolvimento dos estudantes com TEA de acordo com o informante, por meio de teste qui-quadrado. 


\section{Resultados}

Em sua maioria, os cuidadores eram mulheres $(95,2 \%)$, casados $(55,6 \%)$ e do lar $(60,3 \%)$. Responderam também que ter um filho com TEA os impossibilitava de trabalhar formalmente $(61,9 \%)$. A idade média foi de 38,8 anos $(\mathrm{DP}=7,93)$. Notou-se ainda que $38,1 \%$ dos pais/cuidadores tinham o Ensino Médio completo, 17,5\% o Ensino Fundamental incompleto e 12,7\% concluíram o nível Superior.

Em relação aos 51 professores, a idade média foi de 35,11 anos $(\mathrm{DP}=7,06)$ e, em sua maioria, eram do sexo masculino (56,9\%). Acerca da escolaridade, $21(41,2 \%)$ tinham pós-graduação completa, 15 pós-graduação incompleta $(29,4 \%), 13(25,5 \%)$ graduação completa e dois $(3,9 \%)$ professores tinham graduação incompleta. A maioria dos professores era experiente quanto a sua atuação no ambiente escolar, sendo que 34 $(66,7 \%)$ tinham mais de cinco anos de formação acadêmica. Destaca-se que somente três (3,9\%) professores declararam ter realizado cursos na área da Educação Especial, porém nenhum era direcionado ao ensino de crianças com TEA.

Dentre os 19 alunos com TEA participantes, 18 eram do sexo masculino. Em relação à idade, cinco alunos tinham 10 anos de idade, quatro 12 anos, três 13 anos, três 14 anos e um aluno tinha 15 anos.

Pais/cuidadores, professores e estudantes com TEA, em geral, não mencionaram, de forma expressiva, os aspectos mais relevantes da definição de bullying (repetição, diferença de poder e intencionalidade), conforme Tabela 1. No que concerne ao aspecto repetição e diferença de poder, teve uma maior porcentagem de professores que as mencionaram em relação à porcentagem de pais e alunos, sendo essas diferenças estatisticamente signifıcativas.

Tabela 1 - Definição de bullying segundo pais/cuidador, professor e estudante com TEA (Fortaleza/ CE - 2016)

\begin{tabular}{|c|c|c|c|c|c|}
\hline & \multicolumn{3}{|c|}{ Informantes (\%) } & \multirow[b]{2}{*}{ X2 (g.l) } & \multirow[b]{2}{*}{$p$} \\
\hline & Cuidador & Professor & Estudante & & \\
\hline Mencionou & $\mathrm{n}=63$ & $\mathrm{n}=51$ & $n=19$ & & \\
\hline \multicolumn{6}{|l|}{ Repetição: } \\
\hline Sim & $3,4(2)$ & $25,5(13)$ & 0 & \multirow{2}{*}{$15,73(2)$} & $<0.001$ \\
\hline Não & $96,6(57)$ & $74,5(38)$ & $100(19)$ & & \\
\hline \multicolumn{6}{|l|}{ Modalidade: } \\
\hline Agressão Física & $8,5(5)$ & $49,0(25)$ & $26,3(5)$ & \multirow[b]{3}{*}{$41,13(2)$} & \multirow[b]{3}{*}{$<0.001$} \\
\hline Agressão Psicológica & $71,2(42)$ & $43,1(22)$ & $5,3(1)$ & & \\
\hline Combinação & 0 & $7,8(4)$ & $68,4(13)$ & & \\
\hline \multicolumn{6}{|l|}{ Preconceito: } \\
\hline Sim & $57,6(34)$ & $5,9(3)$ & $5,3(1)$ & \multirow{2}{*}{$41,52(2)$} & $<0.001$ \\
\hline Não & $42,4(25)$ & $94,1(48)$ & $94,7(18)$ & & \\
\hline \multicolumn{6}{|l|}{ Diferença de poder: } \\
\hline Sim & $3,4(2)$ & $17,6(9)$ & 0 & \multirow{2}{*}{$9,20(2)$} & $<0.010$ \\
\hline Não & $96,6(57)$ & $82,4(42)$ & $100(19)$ & & \\
\hline
\end{tabular}

Fonte: dados da pesquisa. 
Quanto às modalidades de bullying, houve maior porcentagem de alunos que mencionaram tanto o tipo físico quanto o psicológico em relação à porcentagem de pais e professores, sendo essa desigualdade estatisticamente relevante. Os professores mencionaram mais a modalidade agressão física enquanto pais/cuidadores mais a psicológica. Os cuidadores informaram que as agressões psicológicas foram mais recorrentes, exemplificando-as a partir de xingamentos como: "doidinho", "retardado", "anormal" e "doentes". Salienta-se que os referidos participantes também utilizaram frequentemente a palavra preconceito para a definição de bullying, representando mais de $50 \%$ de diferença em relação a professores e estudantes.

Ressalta-se que 30 pais/cuidadores informaram que não sabiam responder se o filho se envolvia em bullying (47,6\%). Conforme a Tabela 2, existiu maior porcentagem de alunos com TEA que disseram ter se envolvido em bullying do que a porcentagem de pais e de professores desses estudantes que afirmaram o envolvimento em bullying. Notase ainda que foi maior a porcentagem de pais que declararam ter seu filho envolvido no problema do que de professores que afirmaram terem seus alunos com TEA envolvidos.

0 tipo de envolvimento mencionado por estudantes com TEA e seus professores foi enquanto vítima, mas houve pais que mencionaram tanto o envolvimento como vítima/ agressor quanto como agressor. Cabe mencionar também que quatro professores informaram não haver nenhum tipo de bullying na escola em que atuam, independentemente de ser ou não com o aluno com TEA, sendo três professores de escolas particulares e um professor da escola pública.

Tabela 2 - Porcentagem de estudantes com TEA envolvidos em bullying e por tipo de envolvimento de acordo com cuidadores/pais, professor e estudante com TEA (Fortaleza/CE - 2016)

\begin{tabular}{|c|c|c|c|c|c|}
\hline $\begin{array}{l}\text { Envolvimento de estudantes com TEA em situação } \\
\text { de bullying nos últimos seis meses (\%) }\end{array}$ & $\begin{array}{l}\text { Cuidador } \\
\mathrm{n}=33\end{array}$ & $\begin{array}{c}\text { Professor } \\
n=51\end{array}$ & $\begin{array}{c}\text { Estudante } \\
\mathrm{n}=19\end{array}$ & X2 (g.l) & $p$ \\
\hline \multicolumn{6}{|l|}{ Tipo de envolvimento } \\
\hline Agressor & $3(1)$ & 0 & 0 & \multirow{3}{*}{$37,326(6)$} & \multirow{3}{*}{$<0.001$} \\
\hline Vítima & $75,8(25)$ & $47,1(24)$ & $63,2(12)$ & & \\
\hline Vítima/agressor & $21,2(7)$ & 0 & 0 & & \\
\hline Não envolvido & 0 & $52,9(27)$ & $29,4(5)$ & & \\
\hline
\end{tabular}

Fonte: dados da pesquisa.

Esses professores que não mencionaram qualquer forma de bullying em suas escolas assim se justificaram: "Isso de bullying é tudo invenção, não passa de brincadeira de criança."; "Aqui na escola trabalhamos fortemente este tipo de violência, e não permitimos esses tipos de comportamentos, principalmente com as crianças deficientes."; "Ficam dando importância demais pra essas coisas de bullying, não vejo eles fazendo isso não, o que vejo são coisas de meninos que brincam como meninos e depois fica tudo resolvido, 
nada demais."; e "Rapaz, sinceramente não consigo entender porque tanto alarde pra esse assunto, isso é coisa de criança”.

Já quanto aos 12 alunos que mencionaram serem vítimas, sete também foram mencionados por seus cuidadores e oito por seus professores, havendo dois que apenas eles próprios se identificaram como vítimas. Dos 24 alunos que foram mencionados por professores como vítimas, nove não conseguiram responder ao questionário, nove também afirmaram serem vítimas e seis também foram mencionados pelos pais. Já dos 25 alunos mencionados por seus pais como vítimas, 16 não conseguiram responder ao questionário, cinco também afirmaram ser vítimas e quatro também foram mencionados pelos professores.

Os cuidadores, em sua maioria, reconheceram que o bullying pode ocasionar importantes consequências, as quais estão explicitadas na Tabela 3. Dos cuidadores que mencionaram existir envolvimento de seus filhos em bullying, a maioria apontou como principal consequência a "falta de vontade de ir à escola", sendo essa a mais apontada quando o filho era vítima. Para os cuidadores com filhos envolvidos como vítima/agressor, a agressividade foi a consequência mais citada. Mais de 50\% dos pais/cuidadores que mencionaram as crianças com TEA como vítimas indicaram que as crianças se isolam socialmente, no entanto, isso não foi citado pelos cuidadores que relacionaram o filho como vítima/agressor.

Tabela 3 - Consequências às crianças com TEA vítimas e vítimas/agressores de bullying de acordo com seus cuidadores (Fortaleza/CE - 2016)

\begin{tabular}{|c|c|c|}
\hline Principais consequências (\%) & $\begin{array}{l}\text { Pais/Cuidadores que relataram o filho ser } \\
\qquad \begin{array}{l}n \text { vítima } \\
n=25\end{array}\end{array}$ & $\begin{array}{l}\text { Pais/Cuidadores que relataram o filho ser } \\
\text { vítima/agressor } \\
\qquad \begin{array}{l}n=7\end{array}\end{array}$ \\
\hline Falta de vontade de ir para a escola & $56(14)$ & $57,1(4)$ \\
\hline Isolamento social na escola & $52(13)$ & 0 \\
\hline Agressividade & $40(10)$ & $71,4(5)$ \\
\hline Tristeza & $44(11)$ & $42,9(3)$ \\
\hline Insônia & $20(5)$ & $28,6(2)$ \\
\hline Dor de cabeça & $8(2)$ & $14,3(1)$ \\
\hline Machucados/ ferimentos & $8(2)$ & 0 \\
\hline Urinar na cama durante o sono & $4(1)$ & $14,3(1)$ \\
\hline Diminuição na performance acadêmica & $4(1)$ & $28,6(2)$ \\
\hline Outras consequências & $60(15)$ & $57,1(4)$ \\
\hline
\end{tabular}

Fonte: dados da pesquisa.

Conforme a tabela 3, o item outras consequências foi bastante citado nos dois tipos de envolvimento em bullying. Cabe destacar algumas palavras, ditas pelos cuidadores, que podem identificar esse item, tais como: raiva (3), choro (10), irritação (1) e vergonha (1). Além disso, um cuidador identificou a perda de apetite entre as consequências e 
sete cuidadores informaram ainda que as crianças não conseguem identificar que estão envolvidas e, por isso, não há consequências negativas.

Os professores de EF mencionaram algumas particularidades do aluno com TEA que o predisporia a se envolver em bullying. Conforme a tabela 4, a maioria dos professores informou que o fato dos alunos com TEA terem poucos amigos e se isolarem durante as atividades em grupo facilitariam o envolvimento com bullying. As características falta de empatia e falta de interesse pelas aulas de educação física foram citadas também pelos professores, mas em menor frequência.

Tabela 4 - Particularidades dos estudantes com TEA contribuintes para o envolvimento como vítimas de bullying de acordo com os professores de educação física (Fortaleza/CE - 2016)

\begin{tabular}{l|cc}
\hline Particularidades: & $\vdots$ & $\begin{array}{c}\text { Professores } \\
\mathrm{n}=24\end{array}$ \\
\hline Poucos amigos & $\vdots$ & $43,1(22)$ \\
\hline Isolamento social & $\vdots$ & $35,5(18)$ \\
\hline Falta de empatia & $\vdots$ & $29,1(15)$ \\
\hline Falta de interesse nas aulas de educação física & $\vdots$ & $29,4(15)$ \\
\hline Agressividade & $\vdots$ & $21,6(11)$ \\
\hline Outros & $\vdots$ & $21,6(11)$ \\
\hline
\end{tabular}

Fonte: dados da pesquisa.

Verificaram-se também particularidades que não se encaixaram dentro dos itens pontuados no questionário, sendo: "não falar" (dois professores), "fazer barulhos estranhos, como zumbidos e gritos histéricos" (um professor), "fixação em determinado assunto (garrafas pet)" (um professor) e "agitação motora anormal” (um professor). Todas essas particularidades foram citadas pelos professores como sendo possíveis facilitadores para o envolvimento dos alunos como vítimas de bullying.

\section{Discussão}

Em relação ao perfil dos cuidadores, a pesquisa identificou que a maioria eram mulheres, casadas, do lar, além de terem declarado não trabalhar devido à dedicação exclusiva ao cuidado dos filhos. Esse perfil dos cuidadores é semelhante ao encontrado na metasintese feita por Ooi et al. (2016), que descreve que muitas mães de crianças com TEA tiveram de deixar seus empregos ou abrir mão de oportunidades de carreira para cuidar dos filhos e acompanhá-los no tratamento.

Os professores de EF desta pesquisa, em sua maioria, eram do sexo masculino, com média de idade de 35 anos e com pós-graduação completa ou em curso. Ressalta-se que poucos professores tinham formação específica na área de Educação Especial e Inclusiva. Corroborando esses achados, o IBGE (2010) apontou que a maioria dos professores de EF no Brasil são homens e jovens. Quanto à qualificação específica na área de Educação 
Especial, estudos apontam para a necessidade de que essa ocorra com maior frequência na formação do profissional de EF (FIORINI; MANZINI, 2014).

Em relação aos estudantes com TEA, esses foram predominantemente crianças do sexo masculino, com idade entre dez e doze anos de idade. Os participantes do presente estudo assemelharam-se aos dos estudos de Cappadocia; Weiss; Pepler (2012) e Rieffe et al. (2012) quanto à idade e ao gênero, sendo que nesses também se investigou a relação entre bullying e o TEA. É importante mencionar que a citada faixa etária é muito suscetível ao envolvimento em bullying (CURRIE et al., 2012). Quanto à maioria da amostra de estudantes ser de meninos, destaca-se que o TEA acomete com maior frequência o sexo masculino (LEHNHARD et al., 2011).

Ressalta-se que a maior parte dos estudantes com TEA convidados para a pesquisa não tinha habilidades para compreender às questões do roteiro de entrevista e, por isso, muitos foram excluídos da amostra, sendo essa uma importante limitação do estudo. É desafiador realizar pesquisas com indivíduos com TEA e dar voz a esses sujeitos, conforme também apontaram Hebron; Humphrey; Oldfield (2015). Dessa forma, em estudos que levem em consideração indivíduos com TEA como informantes, torna-se essencial que os pesquisadores construam instrumentos mais adequados às características do transtorno, utilizando-se de múltiplas estratégias para proporcionar a compreensão do fenômeno bullying, como a utilização de vídeos no estudo de Rex; Charlop; Specto (2018) e de cartoons por Altomare et al. (2017).

Quando os participantes foram questionados a respeito da compreensão sobre bullying, percebeu-se que cuidadores, professores e alunos conhecem pouco sobre a definição científica do referido fenômeno, a qual tem como base as seguintes características: intencionalidade, repetitividade e desequilíbrio de poder (OLWEUS, 2013). Esse conhecimento restrito dos informantes destoa do contexto atual de frequente discussão sobre bullying, tanto no meio acadêmico quanto na mídia e no ambiente das escolas. Outra problemática reporta-se ao fato de que se os indivíduos não conhecem as características básicas do bullying, dificilmente o identificarão na prática e buscarão enfrentá-lo (WILLIAMS; STELKO-PEREIRA, 2013).

Contudo, destaca-se que os professores de EF demonstraram maior entendimento sobre bullying do que pais e estudantes. Essa situação pode estar relacionada a um maior acesso que os professores teriam a discussões sobre bullying, seja no seu percurso acadêmico ou em formações nas escolas onde trabalham. Schroeder et al. (2014) afırmaram, em seu estudo de revisão, que intervenções com a participação de professores são efetivas para diminuir o bullying. Porém, devem ir além da discussão sobre definição do fenômeno e suas principais características. Assim, devem incluir estratégias de supervisão do pátio e de outros espaços de recreação, de mediação de conflitos, de detecção e abordagem do bullying e de planejamento de aulas que promovam relações interpessoais mais saudáveis.

Quanto ao pouco conhecimento dos cuidadores a respeito do bullying, o estudo de Floria (2015) também identificou a dificuldade dos pais em definirem o fenômeno. Os pais/cuidadores da presente pesquisa explicaram o bullying como sendo uma prática preconceituosa e discriminatória, indicando que se caracteriza por todos os tipos de violência, inclusive a institucional, com consequências negativas para aqueles que dela 
sofrem. Essa forma de explicação pode estar associada ao contexto de luta pelos direitos das pessoas com TEA.

Quanto à percepção dos alunos com TEA acerca da definição de bullying, percebeuse que buscaram responder ao questionário exemplificando com alguma situação que vivenciaram na escola, ou seja, de maneira mais concreta do que abstrata, o que é peculiar ao TEA. A maior parte associou à definição do bullying às manifestações diretas, não citando os fatores como repetição e diferença de poder, o que se assemelhou a pesquisas internacionais (CAPPADOCIA; WEISS; PEPLER, 2012; CARTER, 2009; KOWALSKI; FEDINA, 2011).

Em referência às modalidades de bullying, houve maior porcentagem de estudantes que mencionaram tanto o tipo físico quanto o psicológico, em comparação à porcentagem de pais e professores. Isso retrata que os estudantes têm uma percepção mais ampla das práticas de bullying, pois podem estar diretamente envolvidos, seja como vítima, autor, autor-vítima ou testemunha. Hwang et al. (2017) discutem que o autorrelato para identificar o bullying tem as vantagens de avaliar diretamente as experiências, sentimentos e consequências dos envolvidos. Porém, a confiança nos autorrelatos pode ser afetada por diferenças na interpretação do bullying, pela capacidade de precisão em reconstruir eventos e pela vontade de relatar experiências dolorosas. Assim, os autores também apontam que crianças que têm dificuldade de interpretar as situações sociais ou as interpretam como ambíguas, devido às suas características comportamentais ou da própria situação, são mais propensas a defınir como bullying eventos que não se enquadram na definição do fenômeno. Dessa forma, o autorrelato constitui-se como importante fonte de informação, mas deve ser analisado com cautela.

Notou-se que os informantes professores mencionaram a modalidade agressão física com maior frequência, enquanto pais e cuidadores citaram mais a psicológica. A agressão física é uma modalidade de bullying de fácil identificação, pois os danos ocasionados, corporais e materiais, são mais visíveis e incontestáveis (RETTEW; PAWLOWSKI, 2016), o que pode explicar o porquê de ter sido a categoria mais citada pelos professores. Além disso, a identificação do bullying físico é provavelmente mais comum no contexto das aulas de EF, já que essas trabalham com práticas corporais, jogos competitivos e, na maioria das vezes, com vestimentas que permitem observar se há marcas de violência nos membros superiores e inferiores dos estudantes (O'CONNOR; GRABER, 2014). Já a agressão psicológica é uma modalidade menos visível, a qual corresponde a humilhar, ameaçar e/ ou ofender a dignidade dos sujeitos (RETTEW; PAWLOWSKI, 2016). Nesse sentido, essa forma de agressão pode ser, por muitas vezes, ignorada pelos adultos de referência.

Percebe-se que as formas de manifestação de bullying identificadas com maior frequência pelos informantes foram as que correspondem ao bullying direto. 0 bullying indireto, por ser mais sutil e não envolver a relação direta entre agressores e vítimas, na maioria das vezes, não é identificado pela comunidade escolar, o que dificulta a interrupção da violência. Destaca-se que o bullying indireto pode ser bem frequente entre crianças e adolescentes com TEA, pois as dificuldades com a comunicação e a interação social tornaos mais vulneráveis a esse tipo de bullying relacional, que se manifesta, principalmente, pela exclusão e pelo isolamento do indivíduo do grupo de pares (SCHROEDER et al., 2014). Outra manifestação de bullying direto que não foi citada pelos participantes foi a agressão 
verbal, a qual é citada como a mais frequente forma de violência sofrida por pessoas com TEA nas revisões (MAÏANO et al., 2016b; SAGGERS et al., 2017; SCHROEDER et al., 2014).

Salienta-se que grande parte dos pais/cuidadores informaram que não sabiam responder se o filho se envolvia em bullying. Esse dado mostra-se preocupante frente ao fato de que os cuidadores são peças-chave para a identificação do bullying, bem como para a intervenção no problema. Nesse sentido, Schroeder et al. (2014) e Shetgiri et al. (2012) indicam que o treinamento de pais para identificar e lidar com bullying é essencial para o enfrentamento desse fenômeno, já que a aprendizagem dessas competências está relacionada a diminuições nas taxas de agressões vivenciadas pelos estudantes nas escolas. Além disso, os pais também são fundamentais para a inclusão escolar de seus filhos com TEA, por meio da sua participação efetiva no cotidiano escolar e no estabelecimento de parcerias e de troca de experiências com os professores (CABRAL; MARIN, 2017).

Em relação à participação em práticas de bullying, houve maior porcentagem de alunos que disseram ter se envolvido em bullying do que a porcentagem de pais e professores que afirmaram o envolvimento em bullying de seus filhos e estudantes. Esse achado pode estar relacionado ao fato de que os estudantes têm uma percepção mais ampla sobre o fenômeno do bullying por estarem envolvidos diretamente (HWANG et al., 2017). Nota-se ainda que foi maior a porcentagem de pais que declararam terem seus filhos envolvidos em bullying do que de professores que afirmaram terem seus alunos com TEA envolvidos. Esta situação também foi encontrada no estudo de prevalência de Campbell et al. (2017) e na revisão de Schroeder et al. (2014), afirmando que os pais podem estar mais próximos ou atentos ao cotidiano dos filhos do que seus professores.

No que concerne ao tipo de envolvimento, a categoria vítima foi a mais frequente entre as três categorias, porém estudantes e professores mencionaram apenas a categoria vítima, enquanto pais também citaram as categorias agressor e agressor-vítima. Esses resultados condizem com a literatura sobre bullying e o TEA, que confirma que indivíduos com TEA envolvem-se em bullying tanto como vítima quanto como agressores e agressoresvítimas (MAÏANO et al., 2016b; HWANG et al., 2018; SCHROEDER et al., 2014).

Porém, Hwang et al. (2018) discutem que as crianças com TEA são muito menos susceptiveis de estar envolvidas em bullying como perpetradoras e/ou perpetradorasvítimas. Além disso, os autores questionam acerca da correta classificação de uma criança com TEA como autora, já que muitos comportamentos típicos do transtorno podem ser confundidos com agressões intencionais. Outro ponto destacado pelo estudo é que quando há o controle de comorbidades psicopatológicas, como do TDAH, o qual aumenta as chances de indivíduos com TEA serem agressores, o risco para ser agressor ou agressorvítima desaparece. Assim, os pesquisadores concluem que focar a atenção nas crianças com TEA como perpetradoras de bullying parece ser uma distração de muitos outros fatores de risco ao problema, os quais podem ser alvos de intervenção.

Os quatro professores que afirmaram não ter bullying em suas escolas apresentaram discursos que naturalizam e amenizam as práticas de intimidação entre pares, definindo o bullying como "brincadeira" ou "coisa de criança”. Mesmo com a grande discussão sobre o tema nos dias atuais, esses tipos de discurso ainda são bem presentes no imaginário popular e, além de disseminar informações equivocadas sobre o fenômeno, também 
contribuem, mesmo que de forma involuntária, para o desenvolvimento e fortalecimento do bullying no ambiente escolar (WILLIANS; STELKO-PEREIRA, 2013).

Destaca-se que dos 12 alunos que mencionaram serem vítimas, sete também foram mencionados por seus cuidadores e oito por seus professores. Verifica-se então que houve boa concordância dos informantes em relação a esses alunos, o que demonstra também que é válido levar em consideração as informações dos próprios estudantes com TEA para a identificação da ocorrência de bullying (CAMPBELL et al., 2017). Schroeder et al. (2014) identificaram que os indivíduos com TEA, considerados aptos para os estudos, tiveram desempenho comparável ao da população em geral, demonstrando poucos erros em relação à identificação do bullying.

Em relação às consequências do bullying, os cuidadores apontaram com maior frequência a falta de vontade de ir à escola quando os filhos eram vítimas. De modo similar, Adams et al. (2016) demonstraram que o bullying prejudica o desempenho e vivência escolar de estudantes com TEA, pois eles passam a se sentir inseguros na escola e a parear o ambiente da escola com os sentimentos negativos ocasionados pelas intimidações.

Já para os cuidadores com filhos envolvidos como vitima-agressor, a agressividade foi a consequência mais citada. Schoelder et al. (2014) apontam que crianças com TEA envolvidas em perpetração como autores-vítimas apresentam maiores taxas de dificuldades de regulação emocional. Dessa forma, quando provocadas, elas podem responder de forma claramente agressiva e visível, o que provocaria maior vitimização. Pozzo; Roché; Siverstein (2018) destacam que é importante deixar claro que o TEA não causa violência ou agressividade e que comportamentos violentos em indíviduos com TEA são ocasionados por terceiras variáveis, incluindo controle parental, ambiente familiar, bullying ou comorbidade psiquiátrica.

Outra consequência do bullying bastante citada por cuidadores de vítimas e vítimasautoras foi a tristeza. Corroborando esses achados, Ung et al. (2016) e Schroeder et al. (2014) apontam que a vitimização de crianças com TEA apresenta correlação significativa com: solidão, tristeza, ansiedade, sintomas depressivos, hiperatividade, elevada sensibilidade emocional e déficit de habilidades sociais. A consequência isolamento social foi também mencionada por cuidadores de vítimas. Vale ressaltar que o isolamento social é uma particularidade da própria criança com TEA, mas que pode se agravar diante do bullying. Os cuidadores com filhos envolvidos no bullying como vítima/agressor não citaram o isolamento social como umas das consequências do bullying.

Os professores de EF também mencionaram algumas particularidades do aluno com TEA que o predisporia a se envolver em bullying, que foram ter poucos amigos e o isolamento social. Estas situações são corroboradas por estudos que indicam os fatores de risco para o envolvimento em bullying de crianças com TEA: características do TEA, vulnerabilidade social, déficit em habilidades sociais, pouco apoio social e ausência de amizades (SCHROEDER et al., 2014; SRECKOVIC et al., 2014). Nesse quesito, houve professores que também citaram a falta de empatia e falta de interesse pelas aulas de educação física. O'Connor; Graber (2014) afırmaram que as práticas de bullying são muito frequentes nas aulas de EF e a demonstração de desinteresse pelas aulas pode estar associada ao medo de ser estigmatizado ou ridicularizado frente ao grupo. 
Destaca-se que a literatura na área da educação especial tem demonstrado o importante papel dos professores como mediadores das interações sociais dos estudantes com TEA no ambiente escolar, pois eles podem intervir facilitando as relações desses indivíduos com seus pares (AGRIPINO-RAMOS; LEMOS; SALOMÃO, 2019). Porém, se os educadores negligenciam esse importante papel na inclusão escolar e justificam isso por conta das características intrínsecas do TEA, poderão estar contribuindo para a exclusão dos estudantes com esse transtorno aos espaços de aprendizagem.

\section{Considerações finais}

Este estudo teve como objetivo identificar o envolvimento de estudantes com diagnóstico de TEA em bullying, de acordo com eles mesmos, seus pais e seus professores de EF. Conforme os resultados encontrados, evidencia-se que o público de escolares com TEA é propenso para o envolvimento em bullying, especialmente como vítimas, o que prejudicaria sua saúde e qualidade de vida, bem como sua inclusão escolar. Além disso, o estudo confirma que ainda há um desconhecimento do fenômeno bullying por parte de peças-chave da comunidade escolar, o que dificultaria a identificação do problema e as possíveis intervenções.

Os dados deste estudo revelam que é urgente a necessidade de capacitação e formação sobre o tema bullying para professores, familiares e alunos, especialmente, para os estudantes com TEA, devido à condição de maior vulnerabilidade. Essas capacitações devem precisar o que seria o fenômeno bullying e tratá-lo como um problema interpessoal e complexo, que vai muito além de culpar os autores de bullying. Deve-se reconhecer que existem fatores do indivíduo, da família, da escola e da sociedade que permitem e impulsionam situações em que uma pessoa sofre agressões frequentes e intencionais por parte de seus pares. Mais especificamente, deve-se reconhecer que a prática de bullying às pessoas com TEA é contrária à perspectiva da Educação Inclusiva, desrespeita o que preconiza o PNEEPEI, a Lei 12.764 e, de modo mais amplo, os Direitos Humanos, devendo ser tratado também como um sintoma de uma sociedade desigual, excludente e normalizadora.

Apesar da relevância deste estudo, é preciso apresentar algumas de suas limitações. Foram aplicadas apenas entrevistas estruturadas, não tendo sido realizadas entrevistas mais aprofundadas para entender as nuances quanto ao envolvimento do aluno com TEA com bullying e tampouco foram feitas observações do cotidiano escolar desse indivíduo. Além disso, não foram utilizados múltiplos recursos que pudessem facilitar o entendimento do estudante com TEA, como desenhos e vídeos. A amostra foi por conveniência e, mesmo que tivesse englobado quantidade considerável de participantes da instituição especializada, os dados podem não ser generalizáveis para outros contextos, sendo necessários mais estudos.

Ainda que haja limitações, este estudo é um dos poucos a respeito do tema no Brasil. Ademais, as conclusões podem subsidiar a construção de programas educativos em que se precise a definição de bullying, se destaque também a modalidade psicológica do problema, se articulem melhor as percepções de pais, professores e alunos, se reconheçam as consequências do problema e se enfatize a necessidade de, para além de evitar o 
bullying, se fomentar, entre alunos, relações prazerosas, proveitosas e felizes, auxiliandoos inclusive a superar particularidades do TEA.

\section{Referências}

ADAMS, Ryan et al. Peer victimization and educational outcomes in mainstreamed adolescents with Autism Spectrum Disorder (ASD). Journal of Autism and Developmental Disorders, New York, v. 46, n. 11, p. 35573566, 2016.

AGRIPINO-RAMOS, Cibele Shírley; LEMOS, Emellyne Lima de Medeiros; SALOMÃO, Nádia Maria Ribeiro. Vivências escolares e transtorno do espectro autista: 0 que dizem as crianças? Revista Brasileira de Educação Especial, Bauru, v. 25, n. 3, p. 453-468, 2019.

ALTOMARE, Alyssa A. et al. When push comes to shove: how are students with autism spectrum disorder coping with bullying? Canadian Journal of School Psychology, Montreal, v. 32, n. 3-4, p. 209-227, 2017.

BAPTISTA, Claudio Roberto. Política pública, educação especial e escolarização no Brasil. Educação e Pesquisa, São Paulo, v. 45, n. 1, p. 1-19, 2019.

BRASIL. Lei 12.764, de 27 de dezembro de 2012. Institui a Política Nacional de Proteção dos Direitos da Pessoa com Transtorno do Espectro Autista; e altera $0 \S 3^{\circ}$ do art. 98 da Lei $n^{0} 8.112$, de 11 de dezembro de 1990. Diário Oficial [da] República Federativa do Brasil, Brasília, DF, 27 dez. 2012. Disponível em: http://www.planalto.gov.br/ccivil_03/_Ato2011-2014/2012/Lei/L12764.htm. Acesso em: 02 nov. 2019.

BRASIL. Lei de Diretrizes de Bases da Educação Nacional. 2. ed. Brasília, DF: Senado Federal, 2018. Disponível em: https://www2.senado.leg.br/bdst/bitstream/handle/id/544283/lei_de_diretrizes_e_ bases_2ed.pdf. Acesso em: 02 nov. 2019.

BRASIL. Ministério da Educação. Política nacional de educação especial na perspectiva da educação inclusiva. Brasília, DF: MEC, 2008.

CABRAL, Cristiane Soares; MARIN, Angela Helena. Inclusão escolar de crianças com transtorno do espectro autista: uma revisão sistemática da literatura. Educação em Revista, Belo Horizonte, v. 33, n. 33, p. 1-30, 2017.

CAMPBELL, Marilyn et al. Bullying prevalence in students with autism spectrum disorder. Australasian Journal of Special Education, Melbourne, v. 41, n. 2, p. 101-122, 2017.

CAPPADOCIA, M. Catherine; WEISS, Jonathan A.; PEPLER, Depra. Bullying experiences among children and youth with autism spectrum disorders. Journal on Autism and Developmental Disorders, New York, v. 42, n. 2, p. 266-277, 2012.

CARTER, Susan. Bullying of students with asperger syndrome. Issues in Comprehensive Pediatric Nursing, Southampton, v. 32, n. 1, p. 145-154, 2009.

CURRIE, Candace et al. Social determinants of health and wellbeing among young people. Copenhagen: World Health Organization Regional Office for Europe, 2012. 
DEMETRIOU Eleni A. et al. Autism spectrum disorders: a meta-analysis of executive function. Molecular Psychiatry, New York, v. 23, n. 5, p. 1198-1204, 2018.

FIORINI, Maria Luiza Salzano; MANZINI, Eduardo José. Formação do professor de educação física para inclusão de alunos com deficiência. Poíesis Pedagógica, Catalão, v. 12, n. 1, p. 94-109, 2014.

FLORIA, Mariana S. OIhos abertos: a perspectiva de pais sobre bullying. 2015. 141 f. Dissertação (Mestrado em Psicologia) - Universidade Federal de São Carlos, São Carlos, 2015.

GIROTO, Claudia Regina Mosca; POKER, Rosimar Bortolini; VITTA, Fabiana Cristina Frigieri. 10 anos da política nacional de educação especial na perspectiva da educação inclusiva em debate: trajetória, limites e desafios. Revista online de Política e Gestão Educacional, Araraquara, v. 22, n. 2, p. 711-715, 2018. Disponível em: https://periodicos.fclar.unesp.br/rpge/article/view/11968. Acesso em: 21 nov. 2019.

GONZALEZ, Brenda Mendoza; RAMIREZ, Victoria Maldonado. Bullying and social skills in students of basic education. Ciência Ergo-Sum, Ciudad de México, v. 24, n. 2, p. 109-116, 2017.

HEBRON, Judith; HUMPHREY, Neil; OLDFIELD, Jeremy. Vulnerability to bullying of children with autism spectrum conditions in mainstream education: a multi-informant qualitative exploration. Journal of Research in Special Educational Needs, Tamworth, v. 15, n. 3, p. 185-19, 2015.

HOOVER, Daniel W.; KAUFMAN, Joan. Adverse childhood experiences in children with autism spectrum disorder. Current Opinion in Psychiatry, Pittsburgh, v. 31, n. 2, p. 128-132, 2018.

HULLEY, Stephen B. et al. Delineando a pesquisa clínica. 4. ed. Porto Alegre: Artmed, 2015.

HUMPHREY, Neil; HEBRON, Judith. Bullying of children and adolescents with autismo spectrum conditions: a 'state of the field' review. International Journal of Inclusive Education, Abingdon, v. 19, n. 8, p. 845$862,2015$.

HWANG, Soojo et al. Autism spectrum disorder and school bullying: who is the victim? Who is the perpetrator? Journal of Autism and Developmental Disorders, New York, v. 48, n. 1, p. 225-238, 2018.

HWANG, Soojo et al. Discrepancy in perception of bullying experiences and later internalizing and externalizing behavior: A prospective study. Aggressive Behavior, Atlanta, v. 43, n. 5, p. 493-502, 2017.

IBGE. Instituto Brasileiro de Geografia e Estatística. Censo demográfico 2010. [S. I.: s. n.], 2010. Disponível em: https://ww2.ibge.gov.br/home/estatistica/populacao/condicaodevida/indicadoresminimos/suppme/ default_educacao.shtm. Acesso em: 02 nov. 2019.

INEP. Instituto Nacional de Estudos e Pesquisas Educacionais Anísio Teixeira. Consulta matrícula 2018: educação especial. [S. I.]: INEP, 2018. Disponível em: https://inepdata.inep.gov.br/analytics/ saw. dll?Dashboard\&PortalPath=\%2Fshared\%2FIntegra\%C3\%A7\%C3\%A30\%20-\%20Consulta\%20 Matr\%C3\%ADcula\%2F_portal\%2FConsulta\%20Matr\%C3\%ADcula\&Page=Educa\%C3\%A7\%C3\%A 30\%20Especial. Acesso em: 02 nov. 2019. 
KASSAR, Mônica de Carvalho Magalhães; REBELO, Andressa Santos. Abordagens da educação especial no Brasil entre final do século XX e início do século XXI. Revista Brasileira de Educação Especial, Marília, v. 24, n. esp., p. 51-68, 2018.

KASSAR, Mônica de Carvalho Magalhães; REBELO, Andressa Santos; OLIVEIRA, Regina Tereza Cestari de. Embates e disputas na política nacional de educação especial brasileira. Educação e Pesquisa, São Paulo, v. 45, n. 1, p. 1-19, 2019.

KOWALSKI, Robin M.; FEDINA, Cristin. Cyber bullying in ADHD and asperger syndrome populations. Research in Autism Spectrum Disorders, London, v. 5, n. 3, p. 1201-1208, 2011.

LEHNHARDT, Fritiz G. et al. Sex-related cognitive profile in autism spectrum disorders diagnosed late in life: implications for the female autistic phenotype. Journal of Autism and Developmental Disorders, New York, v. 4, n., p. 139-154, 2016.

LOPES, Marcos Venícios de Oliveira. Desenhos de pesquisa em epidemiologia. In: ROUQUAYROL, Maria Zélia; GURGEL, Marcelo (org.). Epidemiologia \& Saúde. 8. ed. Rio de Janeiro: Medbook, 2017. p. 121-132.

MAÏANO, Christophe et al. Prevalence and correlates of bullying perpetration and victimization among school-aged youth with intellectual disabilities: a systematic review. Research in Developmental Disabilities, London, v. 49-50, n. 1, p. 181-195, 2016a.

MAÏANO, Christophe et al. Prevalence of school bullying among youth with autism spectrum disorders: a systematic review and meta-analysis. Autism Research, Kansas City, v. 9, n. 6, p. 601-615, $2016 \mathrm{~b}$.

MELO, Sandra Cordeiro de; SANTIAGO, Mylene Cristina. Alunos com TEA como desencadeadores de processos formativos. Revista online de Política e Gestão Educacional, Araraquara, v. 22, n. esp. 2, p. 890-904, 2018.

O'CONNOR, Jamie A.; GRABER, Kim C. Sixth-grade physical education: an acculturation of bullying and fear. Research Quarterly for Exercise and Sport, Annapolis, v. 85, n. 3, p. 398-408, 2014.

OLWEUS, Dan. School bullying: Development and some important challenges. Annual Review of Clinical Psychology, v. 9, n. 1, p. 751-780, 2013.

00l, Khim Lynn et al. A meta-synthesis on parenting a child with autism. Neuropsychiatric Disease and Treatment, Lexington, v. 12, n. 1, p. 745-762, 2015.

OPAS. Organização Pan-Americana de Saúde; OMS. Organização Mundial da Saúde. Folha informativa transtornos do espectro autista. [S. I.]: OMS, 2017. Disponível em: https://www.paho.org/bra/ index.php?option=com_content\&view=article\&id=5651:folha-informativa-transtornos-do-espectroautista\&ltemid=839. Acesso em: 02 nov. 2019.

PAGNI, Pedro Angelo. Dez anos da PNEEPEl: uma análise pela perspectiva da biopolítica. Educação \& Realidade, Porto Alegre, v. 44, n. 1, p. 1-20, 2019. 
PASIAN, Mara S.; MENDES, Enicéia Gonçalves; CIA, Fabiana. Atendimento educacional especializado: aspectos da formação do professor. Cadernos de Pesquisa, São Paulo, v. 47, n. 165, p. 964-981, 2017.

POZZO, Jill Del; ROCHÉ, Matthew W.; SIVERSTEIN, Steven M. Violent behavior in autism spectrum disorders: Who's at risk? Aggression and Violent Behavior, Fort Lauderdale, v. 39, n. 1, p. 53-60, 2018.

RETTEW, David C.; PAWLOWSKI, Sara. Bullying. Child and Adolescent Psychiatric Clinics of North America, Baltimore, v. 25, n. 1, p. 235-242, 2016.

REX, Catherine; CHARLOP, Marjorie H.; SPECTO, Vicki. Using video modeling as an anti-bullying intervention for children with autism spectrum disorder. Journal of Autism and Developmental Disorders, New York, v. 8, n. 8, p. 1-13, 2018.

RIEFFE, Carolien et al. Don't anger me! Bullying, victimization, and emotion dysregulation in young adolescents with ASD. European Journal of Developmental Psychology, Amsterdam, v. 9, n. 3, p. 351370, 2012.

ROSE, Chad A.; MONDA-AMAYA, Lisa E.; ESPELAGE, Dorothy L. Bullying perpetration and victimization in special education: a review of the literature. Remedial and Special Education, Kansas City, v. 32, n. 2, p. 114-130, 2011.

SAGGERS, Beth et al. Understandings and experiences of bullying: impact on students on the autism spectrum. Australasian Journal of Special Education, Melbourne, v. 41, n. 2, p. 123-140, 2017.

SANTOS, João Otacílio Libardoni dos et al. Atendimento educacional especializado: reflexões sobre a demanda de alunos matriculados e a oferta de salas de recursos multifuncionais na rede municipal de Manaus-AM. Revista Brasileira de Educação Especial, Marília, v. 23, n. 3, p. 409-422, 2017.

SCHROEDER, Jessica $H$. et al. Shedding light on a pervasive problem: a review of research on bullying experiences among children with autism spectrum disorders. Journal of Autism and Developmental Disorders, New York, v. 44, n. 7, p. 1520-1534, 2014.

SHETGIRI, Rashmi et al. Parental characteristics associated with bullying perpetration in US children aged 10 to 17 years. American Journal of Public Health, Washigton, DC, v. 102, n. 12, p. 2280-2286, 2012.

SRECKOVIC, Melissa A.; BRUNSTING, Nelson. C.; ABLE, Harriet. Victimization of students with autism spectrum disorder: a review of prevalence and risk factors. Research in Autism Spectrum Disorders, London, v. 8, n. 9, p. 1155-1172, 2014.

UNG, Danielle et al. The relationship between peer victimization and the psychological characteristics of youth with autism spectrum disorder. Research in Autism Spectrum Disorders, London, v. 32, n. 1, p. 70-79, 2016.

WANG, Xin-Xing; KERY, Rachel; XIONG, Qiaojie. Synaptopathology in autism spectrum disorders: complex effects of synaptic genes on neural circuits. Progress in Neuropsychopharmacology \& Biological Psychiatry, Sherbrooke, v. 84, n. 1, p. 398-415, 2018. 
WILLIAMS, Lúcia Cavalcanti de Albuquerque; STELKO-PEREIRA, Ana Carina. Violência nota zero: como aprimorar as relações na escola. São Carlos: UFSCar, 2013.

Recebido em: 05.12.2018

Revisado em: 22.10.2019

Aprovado em: 18.02.2020

Carla Samya Nogueira Falcão é docente do curso de educação física da Universidade Regional do Cariri (URCA), Mestra em saúde coletiva pela Universidade Estadual do Ceará (UECE), especialista em orientação e mobilidade pelo Instituto Federal de Educação, Ciência e Tecnologia do Ceará (IFCE) e graduada em licenciatura plena em educação física pela Universidade Estadual Vale do Acaraú (UVA).

Ana Carina Stelko-Pereira é psicóloga pela Universidade Federal do Paraná (UFPR), mestra em educação especial e doutora em psicologia pela Universidade Federal de São Carlos (UFSCar) e professora adjunta da Universidade Federal do Paraná (UFPR).

Dayse Lôrrane Gonçalves Alves é psicóloga pela Universidade Federal do Ceará (UFC). É especialista em saúde pública pela Universidade Estadual do Ceará (UECE) e em residência multiprofissional em saúde da família e comunidade pela Escola de Saúde Pública do Ceará (ESP/CE). Mestra em saúde coletiva pela Universidade Estadual do Ceará (UECE). 